\title{
Ribavirin Concentrations, Laboratory Variables, and Clinical Outcomes During Treatment of Hepatitis C Infection with First Generation Direct-Acting Antivirals
}

\author{
Halina Michur ${ }^{1}$, Phillip Morgan ${ }^{1}$, Ivana Carey ${ }^{1}$, Nigel Brown ${ }^{2}$, Nigel Heaton ${ }^{1}$, Michael Heneghan ${ }^{1}$, \\ Kosh Agarwal $^{1}$, John Michael Tredger ${ }^{1}$ \\ ${ }^{1}$ Institute of Liver Studies, King's College Hospital, London, UK \\ ${ }^{2}$ Department of Clinical Chemistry, Wansbeck General Hospital, Ashington, UK
}

Email address:

hmichur@nhs.net (H. Michur)

To cite this article:

Halina Michur, Phillip Morgan, Ivana Carey, Nigel Brown, Nigel Heaton, Michael Heneghan, Kosh Agarwal, John Michael Tredger. Ribavirin Concentrations, Laboratory Variables, and Clinical Outcomes During Treatment of Hepatitis C Infection with First Generation Direct-Acting Antivirals. European Journal of Clinical and Biomedical Sciences. Vol. 5, No. 3, 2019, pp. 51-57.

doi: $10.11648 /$ j.ejcbs.20190503.13

Received: July 12, 2019; Accepted: August 20, 2019; Published: September 9, 2019

\begin{abstract}
In treating hepatitis $\mathrm{C}$ infection, identification of reliable markers predicting virological non-response appear central to improving outcome and to prompting changes in dynamic treatment approaches. The interrelationship of ribavirin (RBV) concentrations and laboratory variables with clinical outcomes was evaluated in HCV patients treated with ribavirin, and with or without early direct acting antivirals (DAA). Correlations between RBV concentration, laboratory variables (haemoglobin, absolute lymphocyte, platelet and neutrophil counts, serum creatinine and hepatitis C viral load) and patients' characteristics associated with sustained virological response (SVR) were investigated using multivariate analysis. The 76 patients studied all received interferon (INF) and RBV, with 37 additionally given Boceprevir or Telaprevir. Significant correlations were noted between week $1 \mathrm{RBV}$ concentration and subsequent total exposure at weeks 2,4 and $12(P<0.0001)$. RBV concentrations in excess of $1.0 \mathrm{mg} / \mathrm{L}$ (week 1) and $2.0 \mathrm{mg} / \mathrm{L}$ (week 2) provided targets for avoiding breakthrough $(P<$ $0.05)$. SVR was greater in patients without cirrhosis $(73.3 \%$ vs. $41.3 \% ; P<0.01)$. All patients with an absolute haemoglobin $(\mathrm{Hb})$ fall $>30 \mathrm{~g} / \mathrm{L}$ (week 8) experienced higher SVR rates $(P<0.03$ ). RBV concentrations above $2.7 \mathrm{mg} / \mathrm{L}$ at week 4 or later increased the likelihood of $\mathrm{Hb}$ falls $>30 \mathrm{~g} / \mathrm{L}$. Six factors were predictive of SVR in univariate analysis, and three in multivariate analysis. There is an association between SVR and absolute lymphocyte count, IL-28B CC genotype, and HCVRNA load fall at week $1(>80 \%)$ or week $2(>90 \%)$ in HCV patients treated with INF/RBV and early DAA.
\end{abstract}

Keywords: Hepatitis C, Antiviral Therapy, Direct-acting Antivirals, Ribavirin, Cirrhosis

\section{Introduction}

Ribavirin [RBV] (1-[(2R,3R,4S,5R)-3,4-dihydroxy-5(hydroxymethyl)oxolan-2-yl]-1H-1,2,4-triazole-3carboxamide) has remained a valuable adjunctive component of hepatitis $\mathrm{C}$ anti-viral therapies despite the shift from interferon-based to direct-acting anti-viral (DAA) regimens $[1,2]$. Inter-individual differences in RBV disposition are well documented and common side-effects, such as anaemia, have been associated with high RBV exposure [3]. Studies have suggested that positive treatment outcomes and adverse events may be associated with RBV concentration thresholds
[4] but contradictory data exist [5], perhaps consistent with its multiple mechanisms of action $[6,7]$. The instability of drug concentrations in collected samples [8] further complicates RBV therapeutic monitoring. Nevertheless, some studies suggest that higher plasma RBV concentrations are associated with a higher rate of sustained virological response (SVR) [9], and that monitoring plasma RBV could be a useful tool to intervene early on in the treatment, allowing necessary dose adjustments to improve tolerability and clinical outcome [10-13]. This study examined the features of RBV exposure and their relationship to RBV doses, laboratory variables and outcomes in order to 
distinguish their impact both on the drug's virological efficacy and its adverse effects.

This work investigated the utility of RBV concentration monitoring within the framework of a compassionate use study with the first generation DAA agents boceprevir (BCV) and telaprevir (TLV). Of the diverse cohort of 76 patients, all received IFN-based therapy with RBV, but 37 also received DAAs. Our aims were (i) to assess the inter-relationship of virological response, laboratory outcomes and plasma ribavirin concentrations in cirrhotic and non-cirrhotic patients and (ii) to identify changes in laboratory markers during ribavirin (and anti-HCV) therapy that might predict sustained virological response.

\section{Methods}

\subsection{Patients and Treatments}

Seventy six HCV infected patients (56 males) were admitted to the study between May 2011 to January 2014. Adult patients ( $>18$ years old) who had a minimum of three ribavirin concentrations during their subsequent treatment course were included in the study.

All received body weight-based interferon and ribavirin therapy, with 37 additionally treated with the DAA agents boceprevir (BCV) or telaprevir (TLV). BCV (800 mg 3 times a day) was started 4 weeks after a dual therapy with peginterferon alpha and ribavirin for 24 to 48 weeks depending on the virus detectability at week 8 , while TLV was given at a dose of $750 \mathrm{mg}$ every 8 hours after meals for the first 12 weeks followed by a dual therapy (PegIFN + RBV) for 12-36 weeks.

\subsection{Laboratory Analyses}

Hepatitis C genotype (RT-PCR, Versant HCV genotype 2.0 Assay) and IL28B status were defined prior to admission. Hepatitis C viral load (COBAS AmpliPrep/COBAS TaqMan HCV quantitative kit), haemoglobin, absolute lymphocyte (ALC), platelet (APC) and neutrophil (ANC) counts and serum creatinine were also measured initially and at routine follow-up visits (weeks 1, 2, 4, 8, 12, 24 and 48). Ribavirin plasma concentrations were measured as described by Brown et al [8]. Concentrations ( $\mathrm{mg} / \mathrm{L}$ plasma) were summated over time using the linear trapezoidal rule to calculate RBV exposure (mg/L x days) between successive measurements.

\subsection{Outcomes}

Clearance of virus (undetectable serum HCV RNA level to limits of detection, $<15 \mathrm{IU} / \mathrm{mL}$ ) were recorded as a rapid virological response (RVR), early viral response (EVR), end of treatment response (ETR), and sustained virological response (SVR).

Multivariate analysis was used to determine the variables independently associated with SVR and forward Wald logistic regression analysis was performed to investigate the likelihood of achieving a SVR. Statistical analysis was performed with GraphPad Prism v7 (7.01) and IBM SPSS v
23. Significance was accorded when $P<0.05$.

\section{Results}

\subsection{Patients and Outcomes}

Table 1 shows a similar range of ages at admission and proportion of males in the DAA $(n=37)$ and non-DAA (IFN+ RBV only) cohorts $(P>0.05$ by contingency analysis). There were 12 naïve and 25 experienced patients who received DAAs and $14 / 25$, respectively who received only IFN+RBV. BCV was administered to 22 and TLV to 15 patients. There was fewer genotype $3(5.9 \%$ vs $22.4 \%$ overall) and more genotype 1 patients (91.9\% vs $69.7 \%$ overall) in the DAA cohort. Forty six (61\%) patients were clinically diagnosed with liver cirrhosis and were graded by their Child - Pugh score [14]. Most patients who received DAA treatment were cirrhotic (73\%). RVR was achieved in $32.9 \%$ of the overall cohort, EVR in $80.3 \%$, ETR in $75 \%$ and SVR in $53.9 \%$. There were no significant differences between the corresponding proportions in the DAA and IFN+RBV only groups $(P>0.05$, Fishers exact test). Breakthrough occurred in $25 \%$ of patients and relapse in $35.5 \%$, with again no significant difference between the DAA / non-DAA groups. Two patients who received DAA treatment and three of non-DAA patients, failed to respond (Table 1).

Table 1. Patient details.

\begin{tabular}{llll}
\hline Variable & DAA & Non-DAA & Total \\
\hline Number & 37 & 39 & 76 \\
Boceprevir & 22 & - & 22 \\
Telaprevir & 15 & - & 15 \\
Age (range in yr) & $42.1-74.0$ & $27.5-78.7$ & $27.5-78.7$ \\
Males & 27 & 29 & 56 \\
Naïve & 12 & 14 & 26 \\
Experienced & 25 & 25 & 50 \\
Cirrhosis & 27 & 19 & 46 \\
Renal Failure & 4 & 8 & 12 \\
Genotype 1 & 34 & 19 & 53 \\
Genotype 2 & 2 & 1 & 3 \\
Genotype 3 & 1 & 17 & 18 \\
Genotype 4 & 0 & 2 & 2 \\
IL28B CC / CT & $13 / 20$ & $13 / 14$ & $26 / 34$ \\
Treatment Response & & & \\
RVR & 13 & 12 & 25 \\
EVR & 32 & 29 & 61 \\
ETR & 30 & 27 & 57 \\
SVR & 20 & 21 & 41 \\
Breakthrough & 10 & 9 & 19 \\
Relapse & 13 & 14 & 27 \\
Non-responders & 2 & & \\
\hline
\end{tabular}

\subsection{RBV Doses, Concentrations and Their Impact on Laboratory Results and Outcomes}

RBV doses were not predictive of early RBV concentrations, with an initial 6.9-fold range of doses leading to a 15.2-fold range in RBV concentrations at week 1. Weak 
correlations between doses and plasma RBV concentrations only achieved statistical significance at week two $(\mathrm{r}=0.258$, $P<0.03$, Spearman rank), but patients $(\mathrm{n}=55)$ treated with high RBV doses ( $\geq 800 \mathrm{mg}$ daily) had higher plasma RBV concentrations at all times; they also achieved an earlier steady state plateau around 8 weeks $v s$. the 12 weeks in the 21 patients treated with lower doses $(\leq 600 \mathrm{mg}$ daily) $(P<$ 0.01, two-way ANOVA). However, high RBV doses were not associated with a greater likelihood of achieving SVR (51\% vs. $57 \%, P=0.798)$ and there were no significant differences in RBV doses or concentrations for patients with and without SVR ( $P>0.13$ by two-way ANOVA). RBV dose reduction during treatment did not impact on the proportion of patients achieving SVR, either in the genotype 1 or genotype 3 cohorts (50\% vs. $56 \%$ (without dose change) and 55\% vs. $55 \%$, respectively).

In the entire cohort, median plasma RBV concentrations rose progressively to $2.8 \mathrm{mg} / \mathrm{L}$ at 8 weeks of treatment before falling to $2.2 \mathrm{mg} / \mathrm{L}$ at 48 weeks (following dose reductions). A strong predictive relationship existed between RBV concentrations at week 1 and subsequent total exposure to the drug at weeks 2, 4, 8 and $12(P<0.0001$ in all cases) (Figure 1). Corresponding Spearman correlation coefficients were $0.986,0.940,0.822$ and 0.777 , respectively.

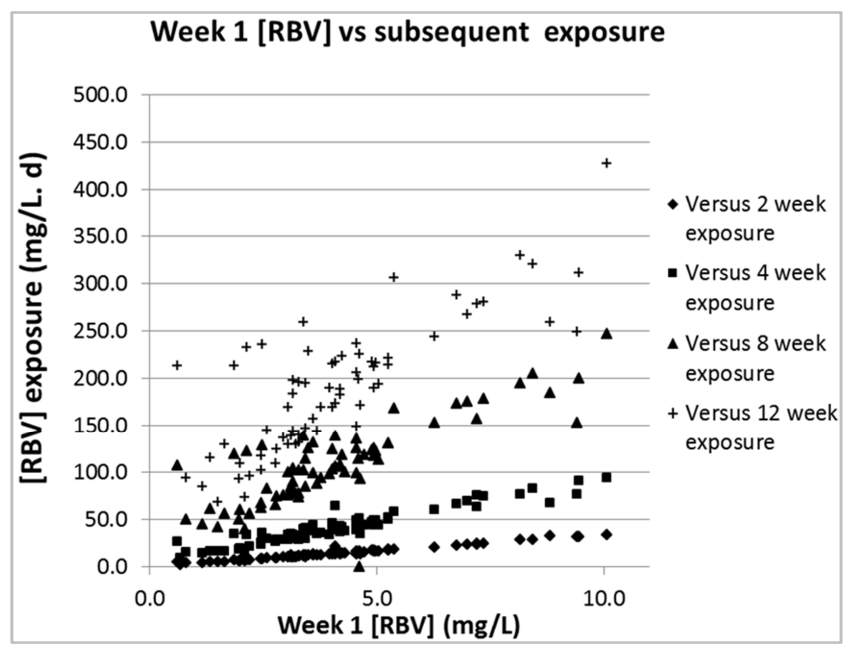

Figure 1. Correlation between $R B V$ concentrations at week 1 and subsequent total exposure during the first 12 weeks of treatment.

Median RBV concentrations (but not doses) differed at weeks 1 and 2 of treatment in patients with and without EVR and ETR $(P<0.05$ for both, Mann Whitney U test). Receiver operating characteristic (ROC) curve analysis for respective discriminant concentrations showed a maximum positive likelihood ratio (sensitivity/(1-specificity)) of 2.86 for EVR and 1.73 for ETR for a RBV concentration of $1 \mathrm{mg} / \mathrm{L}$ at week 1. RBV concentrations were significantly lower and haemoglobin significantly higher at all time points during treatment in patients experiencing breakthrough $(P<0.0001$ and $P<0.001$, respectively by two-way ANOVA). RBV concentrations exceeding $1.0 \mathrm{mg} / \mathrm{L}$ at week 1 and $2.0 \mathrm{mg} / \mathrm{L}$ at week 2 provided targets for avoiding breakthrough $(P<0.05$ by ROC analysis), so providing a possible basis for optimizing $\mathrm{HCV}$ treatment and RBV dosages for improved virological control.

Marked inverse changes in sequential $\mathrm{Hb}$ concentrations and lymphocyte and neutrophil counts paralleled the time course of RBV concentrations: median $\mathrm{Hb}$ fell by a maximum of $33 \mathrm{~g} / \mathrm{L}$ by treatment week 12 , APC by $38 \mathrm{x}$ $10^{9} / \mathrm{L}$ by week 8 , ALC by $0.79 \times 10^{9} / \mathrm{L}$ by week 24 , ANC by $1.57 \times 10^{9} / \mathrm{L}$ by week 24 . Spearman correlation analysis showed a significant inverse association between the entire range of ribavirin concentrations and the corresponding $\mathrm{Hb}$ values $(\mathrm{r}=-0.518)$ and ALC $(\mathrm{r}=-0.297)$, but not ANC $(\mathrm{r}=$ $0.086)$ or APC $(r=0.0495)$. While maximum falls in $\mathrm{Hb}$ and ALC did correlate with RBV exposure, only for $\mathrm{Hb}$ concentrations was there a significant correlation between the fall from baseline and the cumulative RBV exposure at corresponding times to week 12 of treatment $(P<0.01$ in every case, Spearman correlation). Quartile analysis showed a progressive median rise in $\mathrm{RBV}$ exposure for each corresponding $\mathrm{Hb}$ fall at week 12 ( $P=0.067$; KruskalWallis). Back extrapolation suggested that a fall in $\mathrm{Hb}$ greater than $30 \mathrm{~g} / \mathrm{L}$ might be avoided by limiting mean weekly RBV concentrations to $2.7 \mathrm{mg} / \mathrm{L}$ by and beyond 4 weeks of dosing.

Viral load fell rapidly after initiating treatment, with median values of $<0.1 \%$ of baseline achieved by week 2 and undetectable loads before week 8 . The sequential data showed that patients on a high dose RBV regimen (receiving $\geq 800 \mathrm{mg}$ daily) had a more rapid fall in viral load and a greater fall in $\mathrm{Hb}$ concentration (and ALC and ANC) than those treated with the low $(\leq 600 \mathrm{mg})$ daily dose $(P<0.04$, two-way ANOVA in every case). Median serum creatinine concentrations ranged from $77-83 \mu \mathrm{mol} / \mathrm{L}$ without any corresponding temporal relationship to ribavirin concentrations (data not shown).

\subsection{Variables Associated with SVR}

Analysis of the laboratory variables in patients achieving SVR revealed that median ALC and APC began higher and maintained this differential despite falling counts over the treatment course ( $P<0.0001$ for both; two-way ANOVA). However, the fall in ALC was significantly greater in patients who experienced SVR $(P<0.0001)$, while that in APC was not $(P=0.1942)$. Neutrophil counts were lower and neutropenia $\left(\mathrm{ANC}<1500 \times 10^{9} / \mathrm{L}\right)$ more common in those patients not achieving SVR, but neither variable achieved statistical significance (by repeat measures ANOVA and Chi square analysis, respectively). For both ALC and ANC, a common feature was a rise in median counts around or after week 24 for those patients not achieving SVR, while counts in the SVR cohort remained low. Median viral load was lower $(P<0.02$, two-way ANOVA) and the fall in viral load from baseline was faster in patients who achieved SVR $(P<$ 0.02 for the interaction of treatment week and fall in viral load, two-way ANOVA).

Haemoglobin concentration fell progressively during treatment in all patients. Anaemia $(<130 \mathrm{~g} / \mathrm{L}$ in men and $<$ $115 \mathrm{~g} / \mathrm{L}$ in women) developed in $86 \%$ of patients at different times after the initiation of therapy; $17 \%$ of patients 
underwent RBV dosage reductions and $12 \%$ were prescribed erythropoietin (EPO). In the SVR cohort, significantly lower Hb values resulted by week $8(P<0.04$, Mann-Whitney) and there was a more rapid fall $(P<0.01$ for the interaction with time; two-way ANOVA). An absolute fall in $\mathrm{Hb} \geq 30 \mathrm{~g} / \mathrm{L}$ by week 8 was associated with an increased likelihood of SVR (odds ratio 3.26, 95\% confidence intervals $1.27-8.37$ ).

Twenty one of $26(80.8 \%)$ patients with IL28B genotype CC achieved SVR vs. fifteen of 39 (38.5\%) with genotype CT or TT $(P<0.001$ by Chi square analysis). However, antiviral therapy induced similar SVR responses rates in patients stratified by HCV genotype $1(54.7 \%)$ and genotype $3(55.6 \%)$. Although SVR rates were significantly higher for all patients with an absolute $\mathrm{Hb}$ decline $>30 \mathrm{~g} / \mathrm{L}$ at week $8(P$ $<0.03$ by Chi square analysis), there was no difference in SVR rates between patients with HCV Gen 1 (55\%) and Gen $3(55 \%)(P<0.05$ by $C h i$ square analysis $)$.

More treatment-naïve patients achieved SVR than those who were anti-HCV treatment-experienced ( $65 \%$ vs. $48 \%)$, but this failed to achieve significance. However, lymphocyte counts were significantly lower and RBV concentrations significantly higher in retreated patients throughout treatment $(P<0.001$ in both cases, by two-way ANOVA).

Comparison of the DAA and non-DAA cohorts showed that similar proportions achieved SVR (54.1\% vs. 53.8\%), but lymphocyte counts and RBV doses and concentrations were higher during the first 12 weeks of DAA treatment $(P<$ 0.01 by two-way ANOVA in both cases).

Cirrhosis profoundly affected ALC, ANC and APC $(P<$ 0.01 in each case, two-way ANOVA) with baseline and subsequent values all higher in patients with no or mild cirrhosis (Child-Pugh scores of 5-6) than in patients with Child-Pugh scores of 7-8. Viral load in the group of noncirrhotic patients and those with mild cirrhosis was higher at baseline $(P<0.05$, Mann-Whitney), but there was no significant difference in viral load fall between the two groups $(P=0.651$, two-way ANOVA $)$, nor in relation to the severity of cirrhosis $(P>0.05$, Mann-Whitney).

\subsection{Predictors of Response}

The following baseline patient characteristics and ontreatment variables were investigated: age, gender, treatment naïve vs. experienced, cirrhosis, $I L-28 B$ CC vs. CT/TT genotype, HCV genotype 1 vs. 3, RBV concentration, and $\mathrm{RBV}$ dosage, baseline albumin concentration, $\mathrm{Hb}$ level, $\mathrm{Hb}$ fall from baseline, creatinine concentration, ANC, PLC, ALC, ALC fall from baseline, viral load and viral load fall.

To identify the variables independently associated with SVR, multivariate analysis was performed with 6 variables differing significantly by univariate analysis. These included IL28B genotype $(\mathrm{P}<0.05)$, ALC (week 1-12, $P<0.0001)$, APC (week 2-8, $P<0.05$ ), ANC (week $8, P<0.05$ ), $\mathrm{Hb}$ concentration fall (week $8, P<0.03$ ) and viral load fall (week $1-4, P<0.01$ ). The focus was on early indicators of outcome to maximize clinical utility. By forward Wald logistic regression analysis, three variables were associated with a significant early likelihood of achieving a SVR: lymphocyte count (Odds Ratio (OR) at baseline, 2.94 (95\% confidence intervals, $1.33-6.47)$, week $1,10.84(1.90-$ 62.0), week 2, $2.99(1.20-7.43))$; viral load fall (OR week 1, 1.05 (1.01 -1.08), week 2, $1.04(1.00-1.08))$ and $I L-28 B$ CC genotype (baseline OR, $0.179(0.05-0.60)$ (Table 2). The predictive values (binary logistic regression) of absolute lymphocyte count for SVR were within or just below the normal range $\left(1.3 \times 10^{9} / \mathrm{L}-4.0 \times 10^{9} / \mathrm{L}\right)$ i.e., $1.51 \times 10^{9} / \mathrm{L}$ at baseline, $1.34 \times 10^{9} / \mathrm{L}$ at week $1,1.13 \times 10^{9} / \mathrm{L}$ at week 2$)$ and the viral load fall expected would be $80 \%$ at week 1 , and $90 \%$ at week 2 .

Table 2. Factors associated with SVR in patients with HCV by Logistic regression model.

\begin{tabular}{|c|c|c|c|c|c|c|}
\hline \multirow{2}{*}{ Factor } & \multirow{2}{*}{ Univariate analysis } & \multirow{2}{*}{ Week } & \multicolumn{4}{|c|}{ Multivariate analysis } \\
\hline & & & Odds ratio & 95\% Wald confidence & $P$ value & R Square \\
\hline \multirow{3}{*}{$\begin{array}{l}\text { Absolute Lymphocyte } \\
\text { count (ALC) }\end{array}$} & $\mathrm{P}=0.001$ (Mann-Whitney) & Baseline & 2.935 & $1.332-6.467$ & 0.008 & 0.333 \\
\hline & $\mathrm{P}=0.001$ (Mann-Whitney) & Week 1 & 10.843 & $1.896-62.012$ & 0.007 & 0.606 \\
\hline & $\mathrm{P}=0.001$ (Mann-Whitney) & Week 2 & 2.99 & $1.204-7.427$ & 0.018 & 0.436 \\
\hline \multirow{2}{*}{$\begin{array}{l}\text { Viral load } \\
\text { fall }\end{array}$} & $\mathrm{P}=0.004$ (Mann-Whitney) & Week 1 & 1.047 & $1.012-1.084$ & 0.008 & 0.606 \\
\hline & $\mathrm{P}<0.0001$ (Mann-Whitney) & Week 2 & 1.042 & $1.002-1.084$ & 0.039 & 0.436 \\
\hline IL-28B CC v CT/TT & $\mathrm{P}<0.001$ (Chi-Square) & Baseline & 0.179 & $0.053-0.603$ & 0.006 & 0.333 \\
\hline
\end{tabular}

ALC, absolute lymphocyte count; CI, confidence interval;

\section{Discussion}

The usefulness of ribavirin concentration monitoring and its impact on SVR has been controversial, but now it is considered almost redundant. This study demonstrated the inter-relationship of ribavirin concentration with laboratory variables, and the likelihood of achieving an SVR.

Plasma ribavirin concentrations were associated with virological, haematological and laboratory outcomes, but not with SVR, in this cohort of 76 diverse patients treated with various anti-HCV regimens. However, we have identified the initial fall in viral load, IL28B genotype and week 1 lymphocyte counts as factors predicting SVR in this population using logistic regression analysis.

Our observations that RBV concentrations do not predict SVR are in agreement with a previous study [5], although others have reported a benefit to measuring $\mathrm{RBV}$ concentration during or at the end of $\mathrm{HCV}$ treatment $[10,15$, 16]. This study supports that achieving target plasma RBV concentrations of $1 \mathrm{mg} / \mathrm{L}$ at 1 week and $2 \mathrm{mg} / \mathrm{L}$ at week 2 did significantly reduce the likelihood of viral breakthrough. Similarly, significantly higher week 1 and 2 RBV 
concentrations were associated with EVR and ETR.

Like RBV concentrations, RBV doses showed no relationship to SVR in our study, with high $(\geq 800 \mathrm{mg} / \mathrm{d})$ and low $(\leq 600 \mathrm{mg} / \mathrm{d})$ doses equally effective. Previous studies have provided conflicting evidence by indicating that patients infected with HCV genotype 1 respond more favourably at doses of ribavirin that exceed $800 \mathrm{mg} / \mathrm{d}$ [17] or that lower doses may be effective [18]. Lower doses have also been suggested as appropriate for genotype 2 and $3 \mathrm{HCV}$ treatment [19]. We, like Janczewska and colleagues [20], were also unable to demonstrate any impact of RBV dose reduction during treatment on achieving SVR. Patient and treatment heterogeneity may underlie these variable data, but no clear evidence of a dose or concentration relationship to SVR seemingly emerges from our studies. Although there was no RBV-untreated cohort in our study to assist interpretation, it seems possible that the adjunctive role of $\mathrm{RBV}$ in the range of anti-HCV viral therapies used may limit any strong relationship of the drug's doses or concentrations to long-term efficacy outcomes.

In contrast, we have presented evidence that RBV concentrations relate to the side-effects of RBV treatment and that cumulative RBV exposure up to 12 weeks was closely related to the drug concentration at week one - long before steady-state had been achieved. This advances the findings of Slavenburg et al [21] who demonstrated that plasma RBV concentrations at weeks two and four correlated with steady-state concentrations at week 8 or 12 . The early prediction of steady state concentrations may be valuable for making early dose adjustments in response to perceived over or under exposure and avoidance of adverse effects which are particularly evident in patients with advanced fibrosis or cirrhosis [22]. Viral load, haemoglobin concentrations and absolute lymphocyte, neutrophil and platelet counts all fell with rising RBV concentrations. The falls in $\mathrm{Hb}$ and ALC were greater at higher RBV exposure but only with $\mathrm{Hb}$ falls did interquartile ranges of both progressively increase in parallel, suggesting the strongest association. A target RBV concentration of $2.7 \mathrm{mg} / \mathrm{L}$ was defined below which falls in $\mathrm{Hb}$ were $<30 \mathrm{~g} / \mathrm{L}$. The haematological changes above are consistent with evidence that the phosphorylated metabolites of RBV accumulate extensively in blood cells, particularly erythrocytes, where they induce oxidative stress in cell membranes and stimulate eryptosis [22]. The impact of additional anti-viral treatments (especially interferon) upon these variables via suppression of haematopoiesis is an alternative contributor, although studies in interferon-free regimens also indicate that ribavirin decreases ALC [23, 24]. The very process of successful viral clearance is another possible contributory cause. The greater fall in $\mathrm{Hb}$ and ALC during treatment, paralleling the faster fall in viral counts, were all features associated here with evolving SVR. The former endorses evidence that either anaemia [25] or a $>30 \%$ fall in $\mathrm{Hb}$ concentrations [26, 27] were associated with in increased likelihood of SVR.

Predictive factors are key in patient management and in achievement of SVR, particularly in patients with unfavourable baseline and on-treatment characteristics. Of the three terms emerging from our multivariate logistic regression of factors independently predicting SVR, IL28B CC genotype has been widely identified and shown particularly influential during DAA therapy [28]. In contrast to IL28B genotype, absolute lymphocyte counts and the fall in viral load are both continuous variables determined by the patient history and response - particularly at week 1 when these data were particularly influential in our model. Several previous reports suggest that rapid virological response or viral response within two weeks of starting treatment are stronger predictors of $\operatorname{SVR}[29,30]$ than even a $\geq 2-\log _{10}$ decrease in HCV RNA, low-level detectable HCV RNA at week 4 or an undetectable level by week 12 of therapy [19, $31,32]$. The current study indicates that a viral load fall of either $80 \%$ at week 1 , or $90 \%$ at week 2 appears to be a better predictor of response than viral load itself. Absolute lymphocyte counts within the normal range at baseline and week 1 of treatment were similarly predictors of SVR. A safe means of treating lymphocytopaenia before starting anti-viral treatment may be beneficial to outcome, particularly in patients with cirrhosis where baseline ALC are low and hepatitis $\mathrm{C}$ antiviral therapy is challenging and will reduce counts further.

\section{Conclusion}

This study demonstrated that absolute lymphocyte count, IL-28B polymorphism, and HCV-RNA viral load fall are more likely to be used as independent markers for sustained virological response in patients with chronic $\mathrm{HCV}$, treated with pegylated interferon, ribavirin with or without first generation DAAs. RBV concentrations do relate to major adverse effects and a maximum target concentration (2.7 $\mathrm{mg} / \mathrm{L}$ at 2 weeks) was defined for their minimisation.

Several regimens with new DAAs have been introduced that showed higher efficacy and optimal safety profile compared to IFN - based treatment. Nonetheless, ribavirin continues to be used to optimise the treatment response of some subgroups of patients, particularly those that have been considered the most difficult to cure. In such patient's analysis of the predictive ability of absolute lymphocyte count and viral load fall for clinical outcome with new regimens and ribavirin may warrant further studies.

\section{Authors' Contributions}

JMT and HM were involved in the design of the study and interpretation of the results. HM - collated the data and wrote the manuscript. IC was involved in interpreting the data, $\mathrm{NH}$, $\mathrm{MH}$ and $\mathrm{KA}$ - critically reviewed the manuscript. PM and NB - developed the ribavirin LC-MS/MS method and critically reviewed the manuscript. JMT - guided the study and critically reviewed/edited the manuscript. All authors reviewed and edited the manuscript and approved the final version. 


\section{Competing Interests}

The authors declare that they have no competing interests.

\section{Availability of Data and Materials}

Data and materials related to this study are available upon request.

\section{Consent for Publication}

Not applicable

\section{Ethics Approval and Consent to Participate}

This is a follow on study based on the previous clinical trial performed in this Institute that had ethical approval.

\section{References}

[1] Feld JJ, Jacobson IM, Sulkowski MS, Poordad F, et al. Ribavirin revisited in the era of direct- acting antiviral therapy for hepatitis C virus infection. Liver Int. 2017; 37 (1): 5-18.

[2] Falade-Nwulia O, Suarez-Cuervo C, Nelson DR, et al. Oral direct-acting agent therapy for hepatitis $\mathrm{C}$ virus infection: $\mathrm{A}$ systematic review. Ann Intern Med. 2017; 166 (9): 637-48.

[3] Karasawa T, Saito T, Ueno Y, et al. Metabolome analysis of erythrocytes from patients with chronic hepatitis $\mathrm{C}$ reveals the etiology of ribavirin-induced hemolysis. Int J Med Sci. 2013; 10 (11): 1575-7.

[4] Morello J, Rodríguez-Novoa S, Jiménez-Nácher I, et al. Usefulness of monitoring ribavirin plasma concentrations to improve treatment response in patients with chronic hepatitis C. J Antimicrob Chemother. 2008; 62 (6): 1174-80.

[5] Kovari H, Russmann S, Ledergerber B, et al. Ribavirin concentrations do not predict sustained virological response in HIV/HCV- coinfected patients treated with ribavirin and pegylated interferon in the Swiss HIV cohort study. PLoS One. 2015; 10 (7): e0133879.

[6] Mondelli MU. The multifaceted functions of ribavirin: antiviral, immunomodulator, or both? Hepatology. 2014; 60 (4): 1126-9.

[7] Werner JM, Serti E, Chepa-Lotrea X, et al. Ribavirin improves the IFN- $\gamma$ response of natural killer cells to IFN-based therapy of hepatitis C virus infection. Hepatology. 2014; 60 (4): 1160-9.

[8] Brown NW, Morgan PE, Agarwal K, et al. Concentration monitoring of plasma ribavirin: Validation of a liquid chromatography-mass spectrometric method and clinical sample collection. Ther Drug Monit. 2016; 38 (1): 50-8.

[9] Lindahl K, Stahle L, Bruchfeld A, et al. High-dose ribavirin in combination with standard dose peginterferon for treatment of patients with chronic hepatitis C. Hepatology. 2005; 41 (2): 275-9.

[10] Kuntzen T, Kuhn S, Kuntzen D, et al. Influence of ribavirin serum levels on outcome of antiviral treatment and anemia in hepatitis C virus infection. PLoS One. 2016; 11 (7): e0158512.

[11] Abenavoli L, Mazza M, Almasio PL. The optimal dose of ribavirin for chronic hepatitis C: From literature evidence to clinical practice: The optimal dose of ribavirin for chronic hepatitis C. Hepat Mon. 2011; 11 (4): 240-6.

[12] Morello J, Soriano V, Barreiro P, et al. Plasma ribavirin trough concentrations at week 4 predict hepatitis $\mathrm{C}$ virus (HCV) relapse in HIV-HCV-coinfected patients treated for chronic hepatitis $\mathrm{C}$. Antimicrob Agents Chemother. 2010; 54 (4): 1647-9.

[13] Pradat P, Virlogeux V, Gagnieu MC, et al. Ribavirin at the era of novel direct antiviral agents for the treatment of hepatitis $\mathrm{C}$ virus infection: Relevance of pharmacological monitoring. Hepat Mon. 2015; 15 (9): 1-5.

[14] Pugh R, Murray-Lyon I, Dawson J. Transection of the oesophagus for bleeding oesophageal varices. Br J Surg 1973; 60: 646-9.

[15] Nakayama M, Kobayashi H, Fukushima K, et al. Predictive factors for 24 weeks sustained virologic response (SVR24) and viral relapse in patients treated with simeprevir plus peginterferon and ribavirin. Hepatol Int. 2016; 10 (1): 15868.

[16] Bodeau S, Durand-Maugard C, Lemaire-Hurtel AS, et al. The end-of-treatment ribavirin concentration predicts hepatitis C virus relapse. Ther Drug Monit. 2013; 35 (6): 791-5.

[17] Conti F, Vukotic R, Lorenzini S, et al. Increase of ribavirin dose improves sustained virological response in $\mathrm{HCV}$ genotype 1 patients with a partial response to peg-interferon and ribavirin. Ann Hepatol. 2014; 13 (2): 196-203.

[18] Reddy KR, Shiffman ML, Morgan TR, et al. Impact of ribavirin dose reductions in hepatitis $\mathrm{C}$ virus genotype 1 patients completing peginterferon alfa-2a/ribavirin treatment. Clin Gastroenterol Hepatol. 2007; 5 (1): 124-9.

[19] Ferenci P, Fried MW, Shiffman ML, et al. Predicting sustained virological responses in chronic hepatitis $\mathrm{C}$ patients treated with peginterferon alfa-2a (40 KD)/ribavirin. J Hepatol. 2005; 43 (3): 425-33.

[20] Janczewska E, Flisiak R, Zarebska-Michaluk D, et al. Effect of peginterferon or ribavirin dosing on efficacy of therapy with telaprevir in treatment-experienced patients with chronic hepatitis $\mathrm{C}$ and advanced liver fibrosis: A multicentre cohort study. Medicine (Baltimore). 2015; 94 (38): e1411.

[21] Slavenburg S, Huntjens-Fleuren HW, Dofferhoff TS, et al. Ribavirin plasma concentration measurements in patients with hepatitis C: early ribavirin concentrations predict steady-state concentrations. Ther Drug Monit. 2011; 33 (1): 40-4.

[22] Oswald G, Alzoubi K, Abed M, et al. Stimulation of suicidal erythrocyte death by ribavirin. Basic Clin Pharmacol Toxicol. 2014; 114 (4): 311-7.

[23] Schmid M, Kreil A, Jessner W, et al. Suppression of haematopoiesis during therapy of chronic hepatitis $\mathrm{C}$ with different interferon alpha mono and combination therapy regimens. Gut. 2005; 54 (7): 1014-20.

[24] Harrington PR, Fleischer R, Connelly SM, et al. Ribavirin reduces absolute lymphocyte counts in hepatitis $\mathrm{C}$ virusinfected patients treated with interferon-free, direct-acting antiviral regimens. Clin Infect Dis. 2015; 61 (6): 974-7. 
[25] Sulkowski MS, Poordad F, Manns MP, et al. Anemia during treatment with peginterferon Alfa-2b/ribavirin and boceprevir: Analysis from the serine protease inhibitor therapy 2 (SPRINT-2) trials. Hepatology. 2013; 57 (3): 974-84.

[26] Sulkowski MS, Shiffman ML, Afdhal NH, et al. Hepatitis C virus treatment-related anaemia is associated with higher sustained virologicresponse rate. Gastroenterology. 2010; 139 (5): 1602-11, 11. e1.

[27] Sievert W, Dore GJ, McCaughan GW, et al. Virological response is associated with decline in hemoglobin concentration during pegylated interferon and ribavirin therapy in hepatitis C virus genotype 1. Hepatology. 2011; 53 (4): 1109-17.

[28] Thompson AJ. Genetic factors and hepatitis C virus infection. Gastroenterology. 2012; 142 (6): 1335-9.
[29] Tamai H, Shimizu R, Shingaki N, et al. Prediction of sustained virological response to telaprevir-based triple therapy using viral response within 2 Weeks. Hepat Res Treat. 2014; 2014: 748935 .

[30] Thompson A, Devine S, Kattan M, et al. Prediction of treatment week eight response \& sustained virologic response in patients treated with boceprevir plus peginterferon alfa and ribavirin. PLoS One. 2014; 9 (8): e103370.

[31] Poordad F, Landaverde C. Rapid virological response to peginterferon alfa and ribavirin treatment of chronic hepatitis $\mathrm{C}$ predicts sustained virological response and relapse in genotype patients. Therap Adv Gastroenterol. 2009; 2 (2): 917.

[32] Sidharthan S, Kohli A, Sims Z, et al. Utility of hepatitis C viral load monitoring on direct-acting antiviral therapy. Clin Infect Dis. 2015; 60 (12): 1743-51. 